\title{
Finite-Element Analysis of Stress on the Proximal Tibia After Unicompartmental Knee Arthroplasty
}

\author{
Mihoro Sano ${ }^{1}$, Yasushi Oshima ${ }^{1}$, Kohei Murase ${ }^{2}$, \\ Katsumi Sasatani ${ }^{1}$ and Shinro Takai ${ }^{1}$ \\ ${ }^{1}$ Department of Orthopaedic Surgery, Nippon Medical School, Tokyo, Japan \\ ${ }^{2}$ Center for Industry-University Collaboration, Graduate School of Engineering Science, Osaka University, Osaka, Japan
}

Background: Because the indications for unicompartmental knee arthroplasty (UKA) are limited, few patients have undergone the procedure. Therefore, it is difficult to decide the acceptable range of variation in the details of UKA on the basis of the available clinical data. The objective of this study was to identify factors that affect the distribution of stress on the proximal tibia after UKA.

Methods: Two-dimensional finite-element analysis of the proximal tibia was used to assess four factors: 1) two types of implants - all ultra-high-molecular-weight polyethylene (UHMWPE) and metal-backed implants, 2) postoperative alignment, 3) coverage of tibial bone, 4) level of the tibial osteotomy.

Results: In cases of varus alignment, high stress values and large areas of deformation were observed on and beneath the implant. In cases of valgus alignment, stress was concentrated at the lateral portion of tibial tray. In comparison with the standard model, stress concentration was greater at the medial edge of the medial condyle in a narrow-coverage model. Stress distribution for the low-osteotomy-level model did not differ markedly differ from that for the standard model. Stress distribution was better for metal-backed implants than for UHMWPE implants.

Conclusions: Proper postoperative alignment must be achieved in UKA. The osteotomy level should be set at the cancellous bone close to the joint line, and preservation of bone stock should be maximized.

(J Nippon Med Sch 2020; 87: 260-267)

Key words: 2-dimensional finite-element analysis (2D-FEA), unicompartmental knee arthroplasty (UKA), tibial implant, alignment

\section{Introduction}

Unicompartmental knee arthroplasty (UKA) is a better procedure than bicompartmental or tricompartmental total knee arthroplasty (TKA) because it preserves normal knee kinematics while maintaining a functional range of motion and preserving bone stock, the anterior and posterior cruciate ligaments, the patello-femoral joint, and the opposite compartment. However, despite these advantages, the efficacy of and indications for UKA are unclear. There were reports of good clinical results during the early development of this procedure ${ }^{1,2}$, but other studies reported less successful results, including deterioration such as implant breakage and loosening ${ }^{3,4}$. These failures result from a number of factors, including poor patient selection, postoperative malalignment, and use of implants with inadequate thickness and no metal backing. In recent years, UKA indications have been strictly controlled and implant designs have been improved, including the use of metal backing ${ }^{5,6}$, and there have been many reports of successful outcomes ${ }^{1,2}$.

The reasons for mechanical failure of UKA were reported to be 1) technical failure, such as varus positioning of the implant and overcorrection of the postoperative leg alignment, 2) poor element design and material, 3) errors in patient selection, such as those with severe varus deformity, involvement of the patello-femoral joint,

Correspondence to Mihoro Sano, MD, Department of Orthopaedic Surgery, Nippon Medical School, 1-1-5 Sendagi, Bunkyo-ku,

Tokyo 113-8603, Japan

E-mail: kohoro1@gmail.com

https://doi.org/10.1272/jnms.JNMS.2020_87-504

Journal Website (https://www.nms.ac.jp/sh/jnms/) 

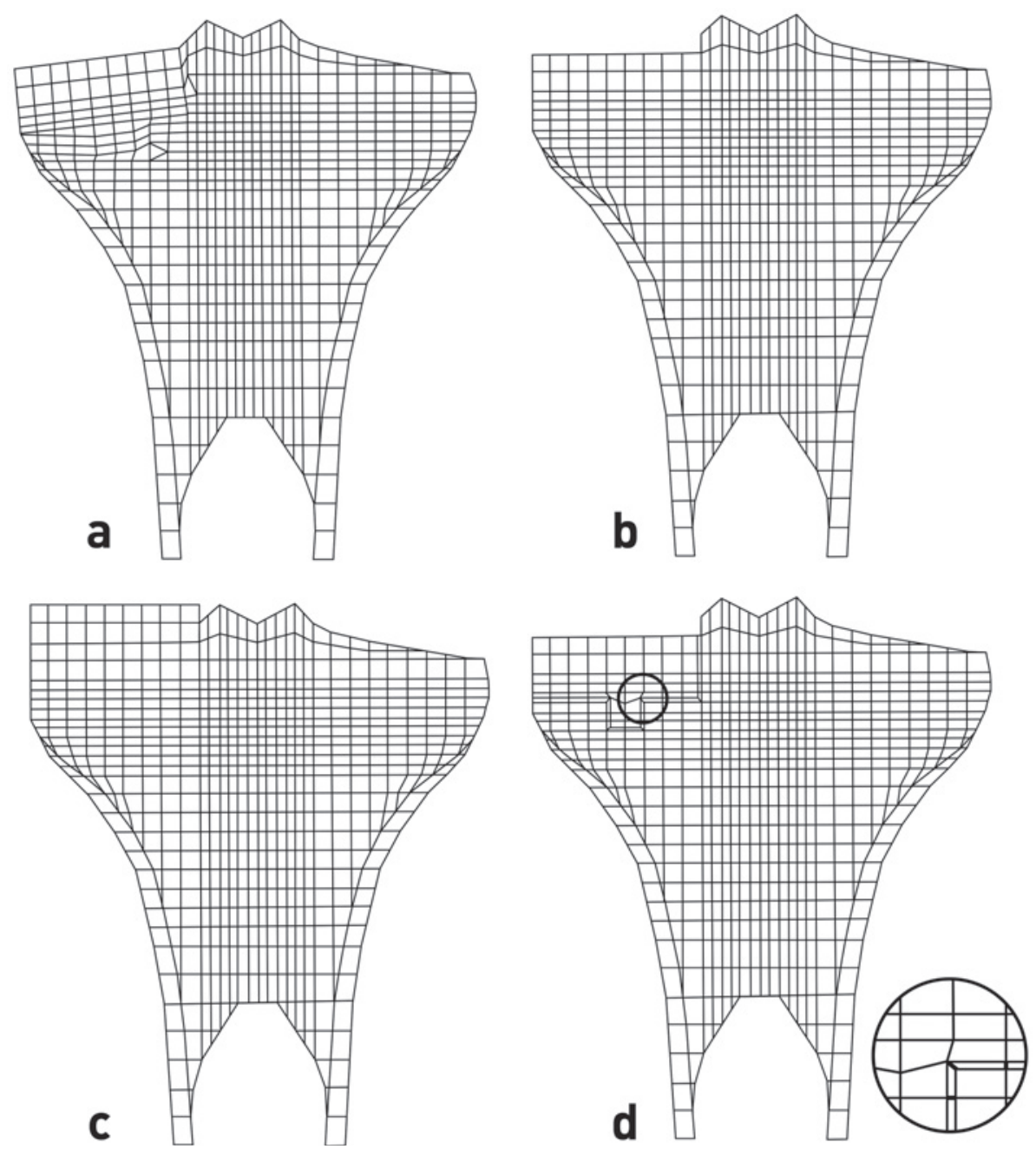

Fig. 1 Finite-element mesh: a) Varus alignment due to undercorrection (688 nodal points, 650 elements); b) Neutral alignment (685 nodal points, 644 elements); c) Valgus alignment due to overcorrection (704 nodal points, 662 elements); d) Nonlinear model using a gap element (neutral alignment, 708 nodal points, 687 elements).

pan-arthritis (including rheumatoid arthritis and chondro-carcinosis), ligament insufficiency, severe obesity, high activity, and osteoporosis. A study of limb alignment in patients undergoing UKA reported positive effects when the mechanical axis was at the center, or slightly medial to the center, of the knee ${ }^{7}$. Many reports cautioned against overcorrection that leads to excess loads in the lateral compartment and early failure ${ }^{8}$. Stress on an implant with inadequate postoperative alignment functions as shear stress and loosens both varus and valgus knees ${ }^{9}$.

Because of the limited number of UKA cases, it is difficult to determine the acceptable alignment range for this procedure from available clinical data. Use of the finiteelement method (FEM) has made structural analysis possible under all conditions by means of computer simulation, and the FEM is often used to optimize the structural design of implants. Advanced application of FEM has been used to analyze artificial joints in orthopedic surgery. Many FEM studies, which have mainly focused on TKA, have shown consistency between simulated results and actual clinical results.

The present study attempted to identify factors that affect stress distribution over the proximal tibia after UKA. To accomplish this, an FEM was used to analyze four elements based on the characteristics of heterogeneous and anisotropic material properties of cancellous bone in proximal tibia ${ }^{10}$, namely, 1) two types of implants: all ultra-high-molecular-weight polyethylene (UHMWPE) and metal-backed implants, 2) postoperative alignment, 3) coverage of tibial bone, and 4) level of the tibial osteotomy. 


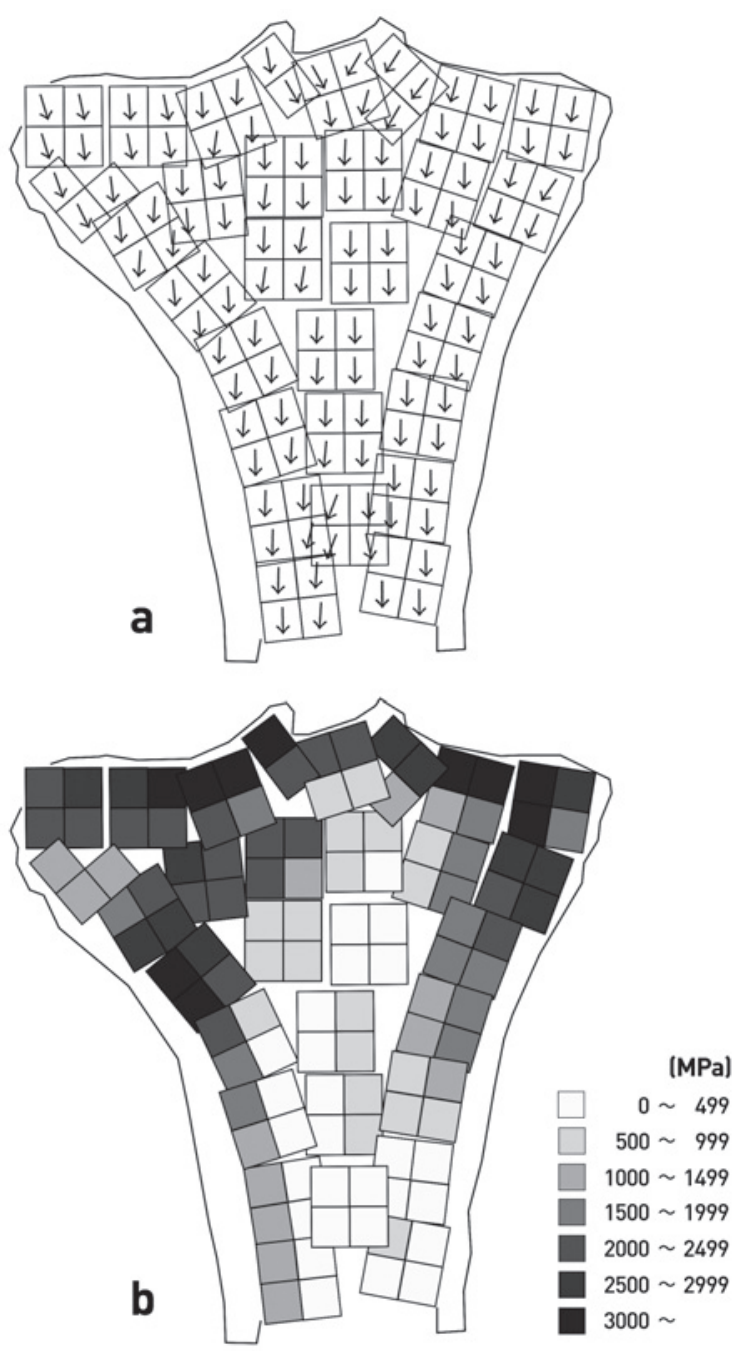

Fig. 2 Mechanical properties of cancellous bone: a) Mean trabecular orientation. b) Distribution of Young modulus values for cancellous bone.

\section{Methods}

To characterize the stress distribution in the proximal tibia under load, a two-dimensional FEM was used to analyze varus alignment with undercorrection (Figure 1 a), neutral alignment (Figure 1b), and valgus alignment with overcorrection (Figure 1c). The morphology of this model was obtained by using data from a previous study ${ }^{8}$.

Each alignment model underwent two types of analysis-linear analysis of the rigid interface of stable implants, and nonlinear analysis (Figure 1d) with application of the gap element in the presence of loosening. Mean trabecular orientation (Figure 2a) and the distribution of Young modulus values in the mean trabecular orientation angle of cancellous bone (Figure $2 \mathbf{b}$ ) were calculated on the basis of numerical analysis of a soft radiograph of a sliced proximal tibia, as previously reported ${ }^{11}$. Figure 3 shows the material properties of cancellous

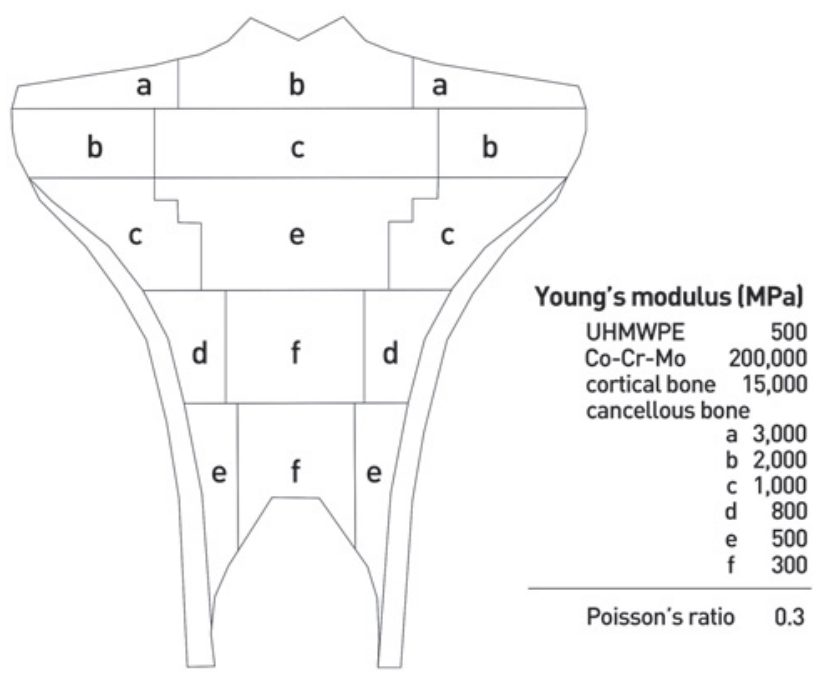

Fig. 3 Material properties of finite-element model.

bone, cortical bone, UHMWPE, and metal (Co-Cr-Mo).

To avoid dispersal of analysis in the nonlinear model, two truss elements were positioned on the medial and lateral sides of the tibia, for model stability (Young modulus, 10-6 MPa). The frictional coefficient of the gap element was 0 , and the Young modulus of the gap element was set at 10-6 MPa. The gap element was set only to transmit compression force. Figure 4 shows the loading and boundary conditions for the model of the proximal tibia after UKA. The load was applied to the neutral alignment; vertical stress over the implant was applied to the varus alignment, and shear stress was applied to the valgus alignment. The load in nonlinear analysis was increased gradually in 10 steps. The distal ends of all models were constrained in the $X-Y$ direction, and the side plate enabling these analyses to consider hoop stress was applied over cortical bone ${ }^{12}$. Cosmos/m (version 2.7) was used for the analysis.

\section{Results}

\section{Stress Distribution in Relation to Postoperative} Alignment

Figure 5 shows the distribution of von Mises equivalent stress values with deformation $(\times 10)$ in linear analysis, and Figure 6 shows the distribution of von Mises equivalent stress values with deformation $(\times 10)$ in nonlinear analysis. Analysis of the varus alignment showed high stress values and large deformations on and beneath implants, which were more obvious for the UHMWPE implants. Analysis of the valgus alignment showed stress concentration at the lateral portion of the tibial tray, which was more obvious in nonlinear analysis. 

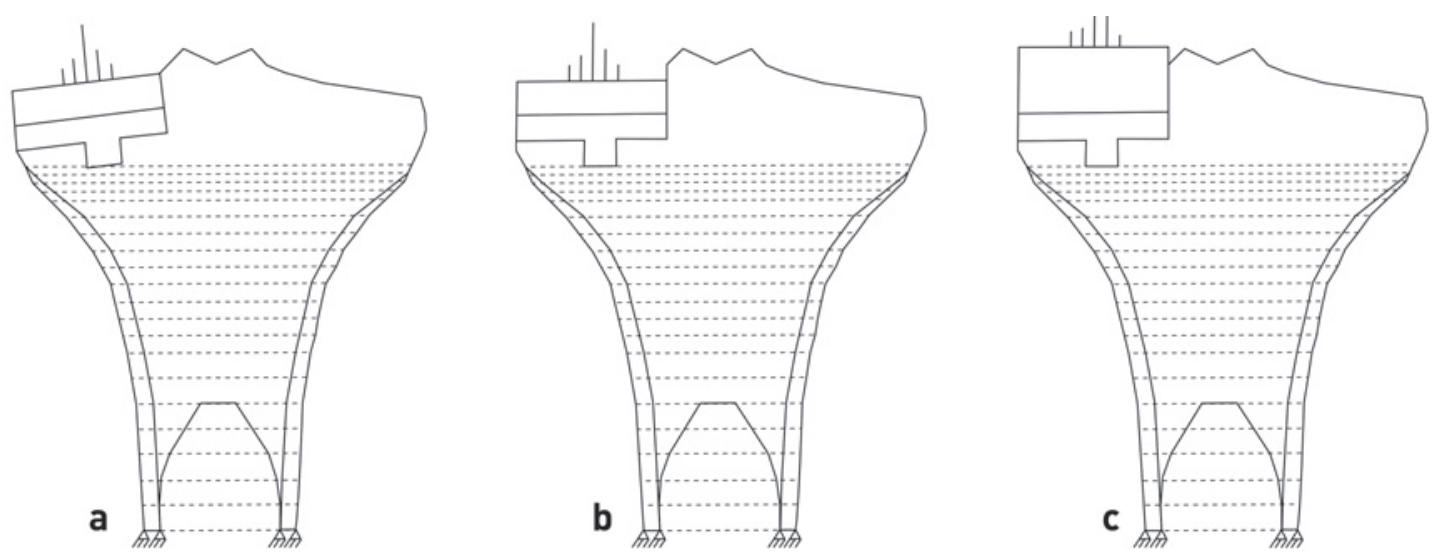

Fig. 4 Loading and boundary conditions: a) Varus alignment; b) Neutral alignment; c) Valgus alignment
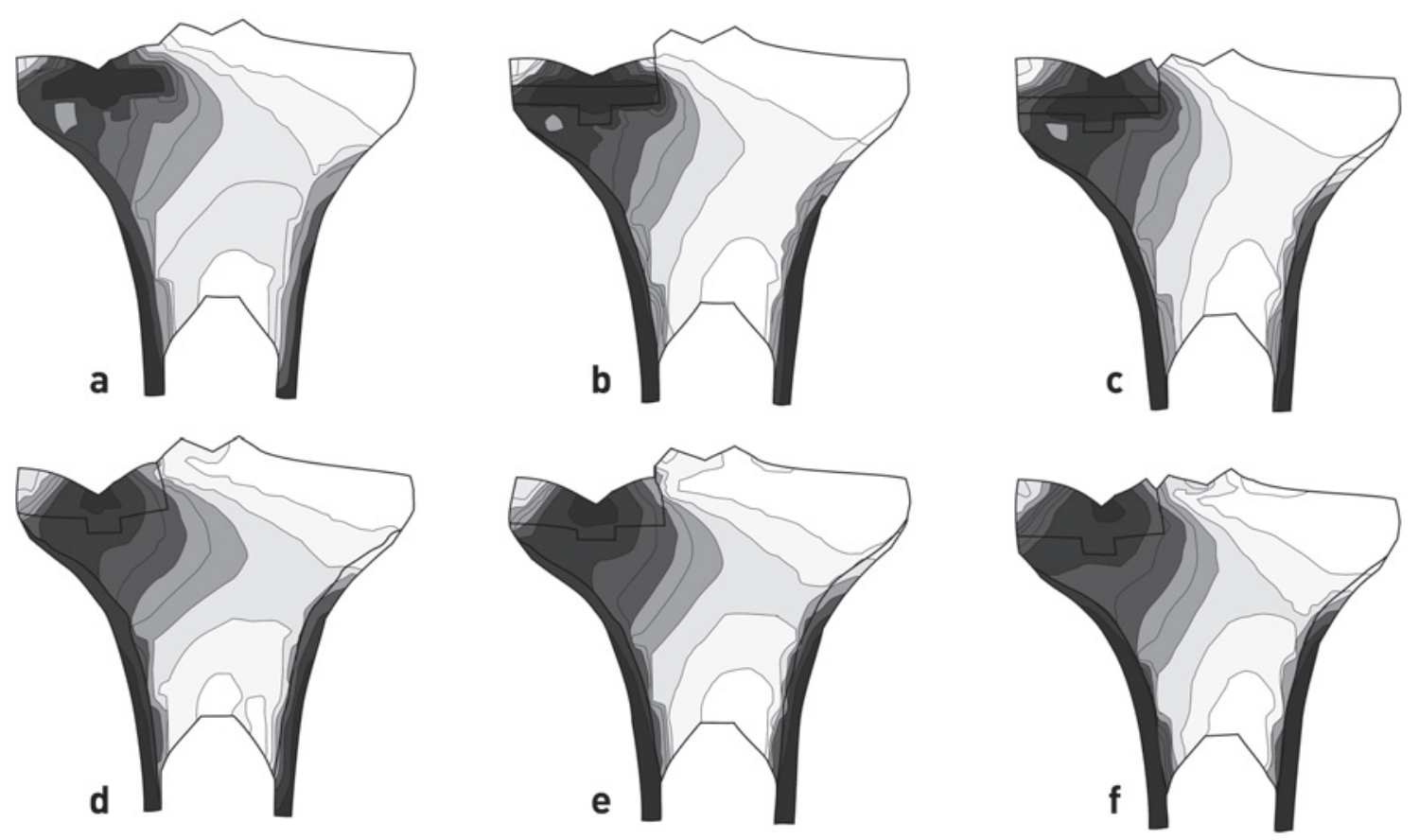

Fig. 5 Distribution of von Mises equivalent stress values in linear analysis with deformation $(\times 10)($ difference in alignment).
a) Metal-backed UKA in varus alignment
b) Metal-backed UKA in neutral alignment
c) Metal-backed UKA in valgus alignment
d) UHMWPE UKA in varus alignment
e) UHMWPE UKA in neutral alignment
f) UHMWPE UKA in valgus alignment

\section{Stress Distribution in Relation to Tibia Coverage}

Figure 7a, d shows the distribution of von Mises equivalent stress values with deformation $(\times 10)$ for narrow coverage of the tibia. As compared with the standard model (Figure $7 \mathbf{b}, \mathbf{e}$ ), the narrow-coverage model showed greater stress concentration at the medial edge of the medial condyle. There was no difference in stress distribution in other areas.
Stress Distribution in Relation to Level of Tibial Osteotomy

Figure $7 \mathrm{c}$, $\mathbf{f}$ shows the distribution of von Mises equivalent stress values with deformation $(\times 10)$ for a low-osteotomy-level UKA. The stress distribution in this model did not substantially differ from that in the standard model.

Evaluation of Cancellous Bone Beneath the Implant

Figure 8 shows von Mises equivalent stress values for 

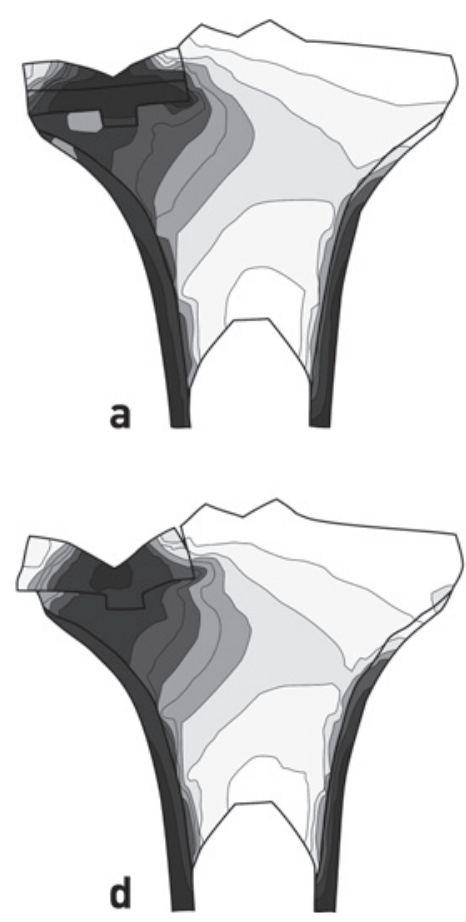
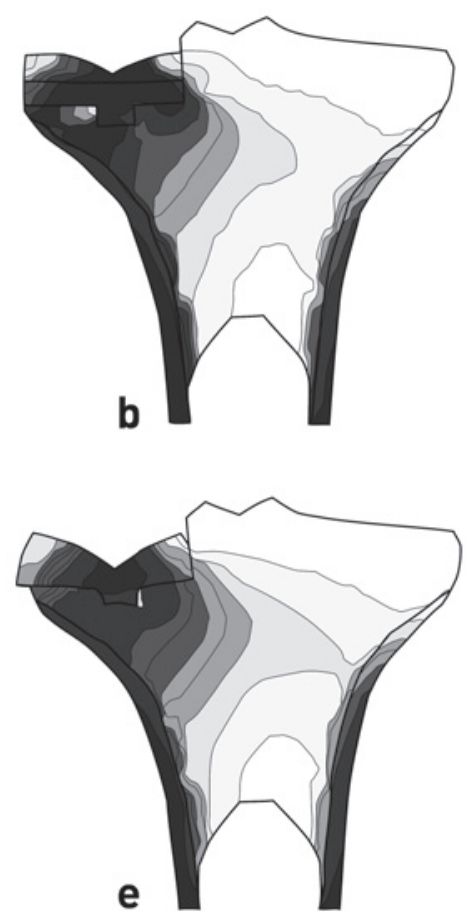

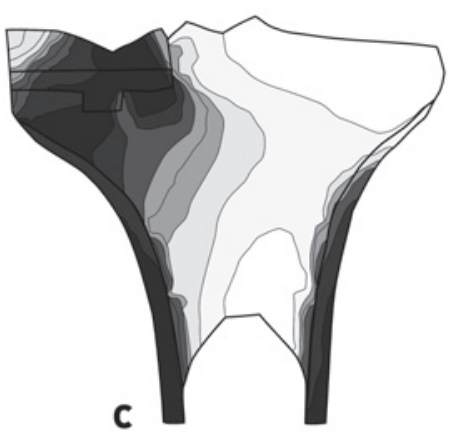

C

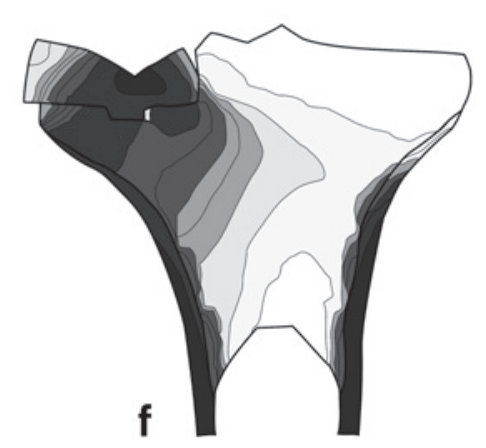

Fig. 6 Distribution of von Mises equivalent stress values in nonlinear analysis with deformation $(\times 10)($ difference in alignment).
a) Metal-backed UKA in varus alignment
b) Metal-backed UKA in neutral alignment
c) Metal-backed UKA in valgus alignment
d) UHMWPE UKA in varus alignment
e) UHMWPE UKA in neutral alignment
f) UHMWPE UKA in valgus alignment

the element in cancellous bone beneath the implant. As compared with the metal-backed implant, the stress concentration was greater around the peg in all alignments in the UHMWPE implant (Figure 8a). In the model with loosening (Figure $\mathbf{8 b}$ ), stress is concentrated at the medial side of the peg and the lateral portion of the tibial tray and stress is lower at the central side of the implant. Stress was high at the medial edge of the medial condyle in the low-osteotomy-level UKA and narrow-coverage UKA.

\section{Discussion}

Finite-element analysis has been used in mechanical analyses of materials and structures since its first use, in the structural analysis of aircraft by Turner et al., in $1956^{10}$. In orthopedics, the FEM was introduced as "a new method to analyze mechanical behavior of skeletal parts" by Brekelmans et al. ${ }^{11}$ FEM has recently been used to examine the causes of prosthetic mechanical problems, such as loosening and sinking of the tibial component of TKA, and in evaluating and optimizing prostheses designs. Studies comparing various designs agree that stress to bone and cement is greater with all-plastic tibial components than with metal-backed components ${ }^{14}$. Many reports have shown that longer central stems decrease stress on proximal trabecular bone $e^{12,15}$, and the results were similar for components with larger surface areas ${ }^{16}$. Clinical results confirm these results of finite-element analysis in TKA. Therefore, the FEM appears to be effective in predicting mechanical issues of implants, the fixation mechanism, and the acceptable range of errors.

Mechanical failure of UKA has been attributed to 1) technical failure, such as varus positioning of the implant and overcorrection of postoperative leg alignment, 2) the design and material of the element, and 3) errors in patient selection, such as those with severe varus deformity, involvement of patello-femoral joint, pan-arthritis (such as rheumatoid arthritis and chondro-carcinosis), ligament insufficiency, severe obesity, high activity, and osteoporosis, among other conditions ${ }^{17}$. In the present study, implant design, material, position, and postoperative alignment were simulated by a FEM and investigated.

Regarding the implant metal backing and thickness, Marmor $^{18}$ and Shurley et al. ${ }^{19}$ reported that a 6-mm tibial 

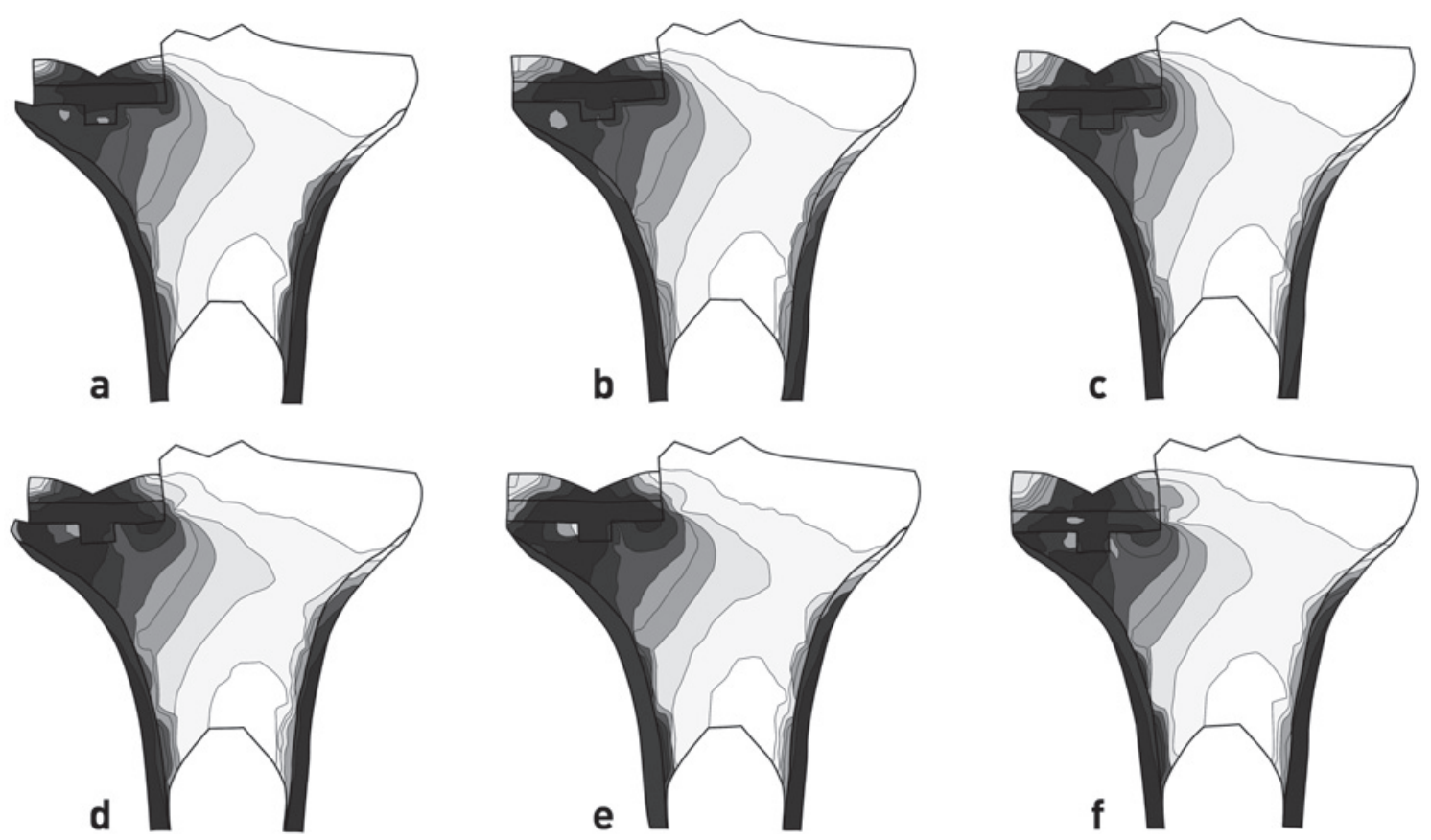

Fig. 7 Distribution of von Mises equivalent stress values in linear and nonlinear analysis with deformation (×10) (difference in coverage and level of osteotomy line).
a) Narrow coverage (linear analysis)
b) Standard (linear analysis)
c) Low-level cutting (linear analysis)
d) Narrow coverage (nonlinear analysis)
e) Standard (nonlinear analysis)
f) Low-level cutting (nonlinear analysis)

component without metal backing tended to result in deformation and loosening. Knutson et al. $^{20}$ reported that plastic deformation of a thin tibial component not only deformed the proximal surface of the component but also introduced eventual deeper deformation. Using roentgen stereophotogrammetric analysis, Ryd et al. observed cold flow in all UHMWPE implants with a thickness of 9 to $12 \mathrm{~mm}$ but not in metal-backed implants ${ }^{21}$. In the present study, metal backing had advantages in stress distribution, as was the case in analyses of TKA.

Patients who underwent UKA had good limb alignment when the mechanical axis was centered. Alignment during UKA is corrected by removing osteophytes, balancing soft tissues, and altering implant thickness. When the implant becomes too tight because of overcorrection, extreme pressure is transmitted to the opposite side of the component, which can result in subluxation. The present findings suggest that stress in underlying cancellous bone is greater with undercorrection than with neutral alignment and that overcorrection concentrates stress at the eminence of the tibia and the underlying bone of the lateral portion of the tibial tray, where bone density is low. The width of the implant is believed to have almost no effect on stress distribution, which was confirmed by finite-element analysis of TKA ${ }^{22}$. Clinically, implant coverage has almost no effect on sinking and loosening in TKA. However, the presence of concentrated stress at the medial edge of the medial condyle in this study suggests that loosening from this area is highly likely.

Severe varus deformity is a contraindication for UKA, as the osteotomy line of the tibia should not reach cortical bone. The present findings indicate that an osteotomy line close to cortical bone would concentrate stress on cancellous bone around the cortical shell. Therefore, preserved bone stock should be maximized in UKA, as it is in TKA.

For models with loosening, stress concentration was observed at the medial side of the peg and lateral region of the tibial tray. This suggests that if loosening occurs, loosening and bone destruction will then extend to other areas. Thus, accuracy in UKA operative procedures and careful consideration of indications are important.

This study has several limitations. There may be many factors that remain to be defined with finite-element analysis. In the present analysis, material connections, such as prosthesis-bone interfaces and UHMWPE-metal 

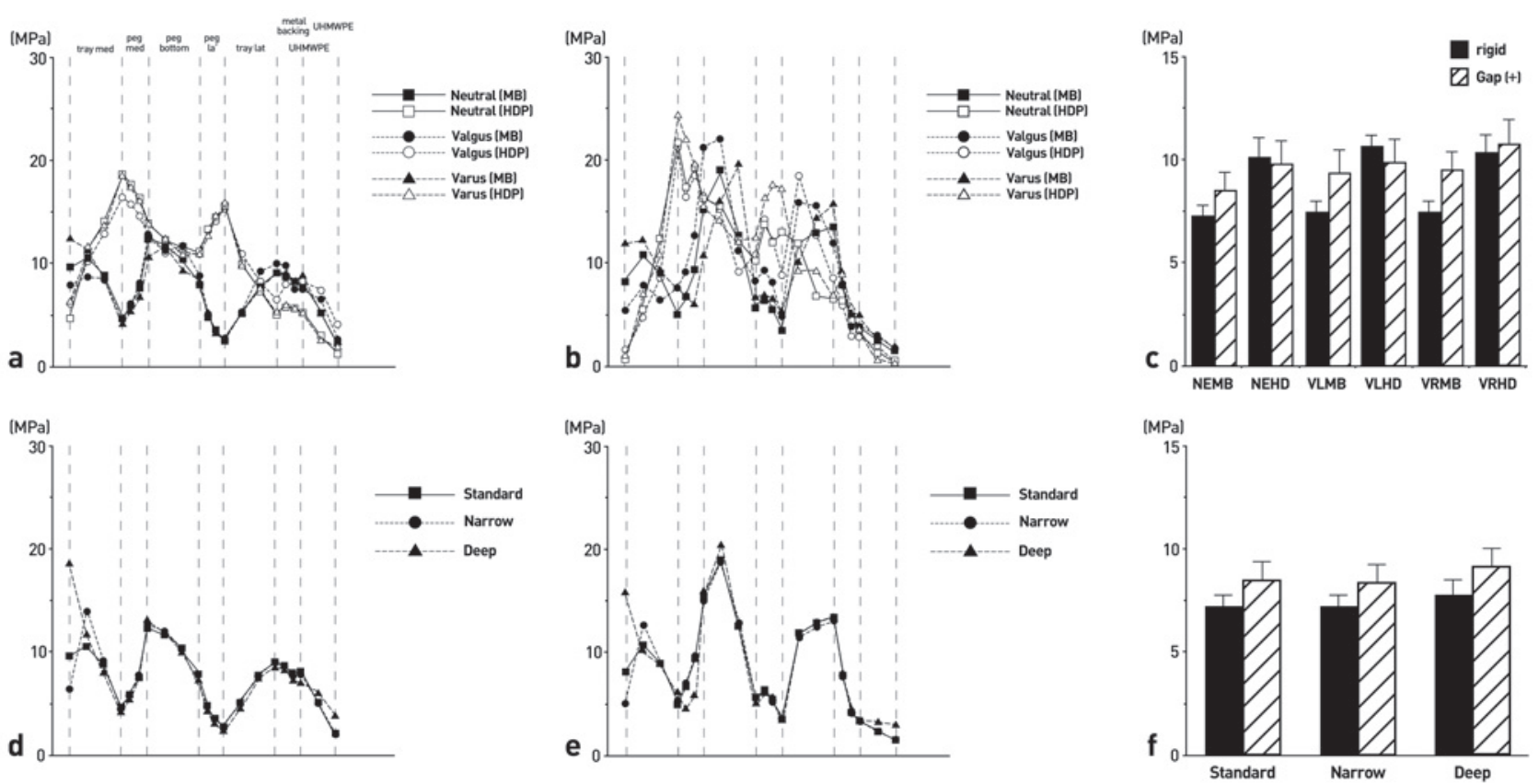

Fig. 8 Distribution of von Mises equivalent stress values in cancellous bone beneath implant.
a) Difference in alignment (linear analysis)
b) Difference in alignment (nonlinear analysis)
c) Difference in alignment (mean $\pm \mathrm{SE}$ )
d) Difference in coverage and level of osteotomy line (linear analysis)
e) Difference in coverage and level of osteotomy line (nonlinear analysis)
f) Difference in coverage and level of osteotomy line (mean $\pm \mathrm{SE}$ )

tray junctions, were assumed to be rigidly bonded. Loading conditions were also defined as static. These conditions are simplifications and not equivalent to actual conditions. Therefore, boundary and loading conditions should be established in future studies. The highly complicated geometry of the knee joint and component design should also be simplified in order to aid in mechanical understanding.

\section{Conclusions}

A two-dimensional finite-element model was used to analyze stress distribution in the proximal tibia after UKA. Stress distribution was better for metal-backed implants than for UHMWPE implants. For UKA, proper postoperative alignment must be achieved. The osteotomy line should be set at cancellous bone close to the joint line with good mechanical properties. In addition, preservation of bone stock should be maximized.

Acknowledgements: We are grateful to Lawrence J. Frumson for checking the English grammar of this manuscript.

Conflict of Interest: The authors declare no competing interests.

\section{References}

1. Berger RA, Meneghini RM, Jacobs JJ, et al. Results of unicompartmental knee arthroplasty at a minimum of ten years of follow-up. J Bone Joint Surg Am. 2005;87:9991006.

2. Winnock de, Grave P, Barbier J, Luyckx T, Ryckaert A, Gunst P, Van den, Daelen L. Outcomes of a fixed-bearing, medial, cemented unicondylar knee arthroplasty design: Survival analysis and functional score of 460 cases. J Arthroplasty. 2018;33:2792-9.

3. Inoue S, Akagi M, Asada S, Mori S, Zaima H, Hashida M. The valgus inclination of the tibial component increases the risk of medial tibial condylar fractures in unicompartmental knee arthroplasty. J Arthroplasty. 2016;31:2025-30.

4. Pandit H, Murray DW, Dodd CA, et al. Medial tibial plateau fracture and Oxford unicompartmental knee. Orthopedics. 2007;30:28-31.

5. Laskin RS. Unicompartmental tibiofemoral resurfacing arthroplasty. J Bone Joint Surg. 1978;60:182-5.

6. Hutt JR, Farhadnia P, Massé V, LaVigne M, Vendittoli PA. A randomised trial of all-polyethylene and metal-backed tibial components in unicompartmental arthroplasty of the knee. Bone Joint J. 97-B(6):786-92.

7. Thornhill TS. Unicompartmental knee arthroplasty. Clin Orthop. 1986;205:121-31.

8. Yoshino N, Inoue N, Watanabe $\mathrm{Y}$, et al. Stress analysis of the proximal tibia after total knee arthroplasty with a finite element method. In: Niwa S, Perren SM, Hattori T, editors. Biomechanics in Orthopedics. Springer Verlag; 1992. p. $253-62$. 
9. Sasatani K, Majima T, Murase K, et al. The optimal alignment of the tibial implant in unicompartmental arthroplasty using 3-dimensional finite analysis. J Nippon Med Sch. 2020;87:60-5.

10. Turner MJ, Clough RW, Martin HC, Topp JL. Stiffness and deflection analysis of complex structures. J Aeronautical Sciences. 1956;23:805-24.

11. Brekelmans WAM, Poort HW, Slooff TJJH. A new method to analyze the mechanical behavior of skeletal parts. Acta Orthop Stand. 1972;43:301-17.

12. Murase K, Crownishield RD, Pedersen DR, Cha ng TS. An analysis of tibial component design in total knee arthroplasty. J Biomech. 1982;16:13-22.

13. Danese I, Pankaj P, Scott CEH. The effect of malalignment on proximal tibial strain in fixed-bearing unicompartmental knee arthroplasty: A comparison between metalbacked and all-polyethylene components using a validated finite element model. Bone Joint Res. 2019;8(2):5564.

14. Scott CE, Eaton MJ, Nutton RW, Wade FA, Evans SL, Pankaj P. Metal-backed versus all-polyethylene unicompartmental knee arthroplasty: Proximal tibial strain in an experimentally validated finite element model. Bone Joint Res. 2017;6(1):22-30.

15. Askew MJ, Lewis JL. Analysis of model variables and fixation post length effects on stresses around a prosthesis in proximal tibia. J Biomech Engng. 1981;103:239-45.

16. Garg A, Walker PS. The effect of the interface on the bone stresses beneath tibial components. J Biomech. 1986;19(2): 957-67.

17. Yoshino N, Takai S, Inoue N, Katayama T. Stress analysis of the proximal tibia after unicompartmental knee arthroplasty with finite-element method. In: Hirasawa Y, Sledge CB, Woo SLY, editors. Clinical Biomechanics and Related
Research. Springer Verlag; 1994. p. 115-23.

18. Marmor L. Unicompartmental knee arthroplasty: Ten- to 13-year follow-up study. Clin Orthop. 1988;226:14-20.

19. Shurley TM, O'Donogue DH, Smith WD, Payne RE, Grana WA. Unicompartmental arthroplasty of the knee. Clin Orthop. 1982;164:236-46.

20. Knutson $\mathrm{K}$, Jonson $\mathrm{G}$, Langer AJ, Larustotti H, Lidgren L. Deformation and loosening of the tibial component in knee arthroplasty with unicompartmental endoprosthesis. Acta Orthop Scand. 1981;52(6):667-73.

21. Ryd L, Lindstrand A, Selvik G. Cold flow reduced by metal backing. Acta Orthop Scand. 1990;61:21-5.

22. Danese I, Pankaj P, Scott CEH. The effect of malalignment on proximal tibial strain in fixed-bearing unicompartmental knee arthroplasty: A comparison between metalbacked and all-polyethylene components using a validated finite element model. Bone Joint Res. 2019;8(2):5564.

(Received, November 19, 2019)

(Accepted, December 25, 2019)

(J-STAGE Advance Publication, January 31, 2020)

Journal of Nippon Medical School has adopted the Creative Commons Attribution-NonCommercial-NoDerivatives 4.0 International License (https://creativecommons.org/licenses/by-nc-nd/4.0/) for this article. The Medical Association of Nippon Medical School remains the copyright holder of all articles. Anyone may download, reuse, copy, reprint, or distribute articles for non-profit purposes under this license, on condition that the authors of the articles are properly credited. 